\title{
Superconducting phase qubit based on the Josephson oscillator with strong anharmonicity
}

\author{
A. B. Zorin ${ }^{1}$ and F. Chiarello ${ }^{2}$ \\ ${ }^{1}$ Physikalisch-Technische Bundesanstalt, Bundesallee 100, D-38116 Braunschweig, Germany and \\ ${ }^{2}$ Istituto di Fotonica e Nanotecnologie, CNR, via Cineto Romano 42, I-00156 Rome, Italy
}

(Dated: August 27, 2009)

\begin{abstract}
We propose a superconducting phase qubit on the basis of the radio-frequency SQUID with the screening parameter value $\beta_{L} \equiv\left(2 \pi / \Phi_{0}\right) L I_{c} \approx 1$, biased by a half flux quantum $\Phi_{e}=\Phi_{0} / 2$. Significant anharmonicity $(>30 \%)$ can be achieved in this system due to the interplay of the cosine Josephson potential and the parabolic magnetic-energy potential that ultimately leads to the quartic polynomial shape of the well. The two lowest eigenstates in this global minimum perfectly suit for the qubit which is insensitive to the charge variable, biased in the optimal point and allows an efficient dispersive readout. Moreover, the transition frequency in this qubit can be tuned within an appreciable range allowing variable qubit-qubit coupling.
\end{abstract}

PACS numbers: 85.25.Dq, 74.50.+r, 03.67.Lx, 03.67.Pp

The superconducting qubits based on the Josephson tunnel junctions (see, e.g., the reviews in Refs. [1, 2]) have already demonstrated their great potential for the quantum computation [3]. The so-called phase qubits present the class of devices which are particularly suitable for integration with microwave on-chip transmission lines and resonators, i.e. the elements which significantly extend the scope of the quantum circuit designs [4]. These qubits are based on the energy quantization in the shallow wells of the inclined cosine Josephson potential [5]. This shape is ensured either by finite bias current $I_{s}$ with the value slightly below the critical current of the Josephson junction $I_{c}$ or a finite flux bias $\Phi_{e}$ applied to the qubit loop (in the case of a loop configuration of the circuit) [6] . In both cases, the energy potential can be approximated by the cubic parabola with a smooth energy barrier isolating the well from one side and allowing escape out of this well enabling a simple readout.

The low depth of the cubic parabola well leads to anharmonicity, viz. successive reduction of the transition energies $\Delta E_{n}=\left(E_{n+1}-E_{n}\right), n=0,1, \ldots$, from bottom to top, necessary for the qubit operation within the basis states $|n=0\rangle$ and $|n=1\rangle$, excluding unwanted excitation of the higher energy states $(n>1)$. Usually the phase qubit is designed such that for appropriate phase bias the cubic potential well includes three-four energy levels with anharmonicity of a few per cent [2, [6]. This is achieved by adjusting the plasma frequency of the Josephson junction both by designing appropriate parameters of the junction and, possibly, by applying external capacitor shunting. The lowering of the energy barrier by applying the socalled measuring pulse, makes possible the reduction of the number of the levels to two $(n=0$ and 1$)$, with notably different rates of escape to a running-phase state (in the case of current bias), or to the lower-energy state in the adjacent well (in the case of the loop configuration of the qubit). The large (but finite) difference of these tunneling rates sets the maximum theoretical value for the fidelity of such measurement to $96.6 \%$. In the carefully designed and optimally biased qubit the best experimental fidelity values approach $90 \%$ [7]. The main disadvantage of such phase qubit is the necessity of resetting it after each measurement.

In contrast to the charge [8], charge-phase [9, 10], flux 11], transmon [12] and recently proposed, the so-called fluxonium [13] qubits, the conventional phase qubits cannot inherently operate in an optimal point, i.e. in the symmetric working point insensitive in the first order to the noise that could give drastic improvement to the qubit performance $[9,14]$. (The exceptions are the recently proposed three-junction interferometer circuit 15 and the so-called camelback potential phase qubit based on the two-junction SQUID [16].) Moreover, the limited anharmonicity of the phase qubit makes the observable reactance impedance (i.e. the Josephson inductance) values in the ground and exited states hardly distinguishable. This poses serious problems for dispersive read-

(a)

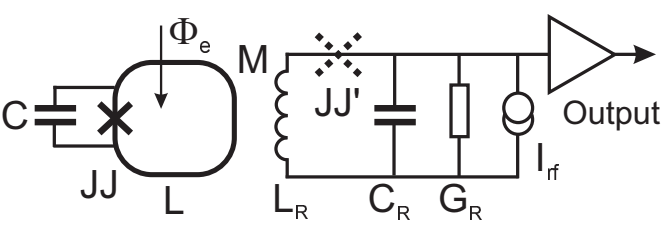

(b)

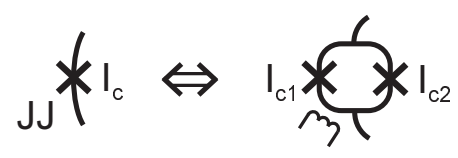

FIG. 1: (a) Electric diagram of the qubit coupled to a resonant circuit and (b) possible equivalent compound (two-junction SQUID) circuit of the Josephson element included into the qubit loop. Capacitance $C$ includes both the self-capacitance of the junction and the external capacitance. Due to inclusion in the resonant circuit of a Josephson junction JJ', the resonator may operate in the nonlinear regime, enabling a bifurcation-based readout. 
out schemes, which proved advantageous where applicable [10, 14, 17] and have allowed quantum nondemolition measurements as well as high fidelity measurements based on bifurcation amplifiers [18, 19].

In this paper we propose an improved phase qubit with significant anharmonicity, in which the manipulation and dispersive readout are both possible in a symmetry point. The circuit diagram of our qubit is shown in Fig. 1a. It comprises the superconducting loop with geometrical inductance $L$ closed by a Josephson junction, generally shunted by an external capacitance, and rf-driven $L_{R} C_{R} G_{R}$ resonance circuit inductively coupled to the qubit loop. The peculiarity of this qubit is the unity value of the SQUID screening parameter $\beta_{L} \equiv\left(2 \pi / \Phi_{0}\right) L I_{c} \approx 1$, where $\Phi_{0}=h / 2 e$ is the flux quantum. This can be achieved by an accurate design of the circuit including replacement of the single junction with a two-junction SQUID, allowing more precise adjustment of the resulting critical current $I_{c}$ (see Fig. 1b).

The potential energy of the stand-alone qubit biased by external magnetic flux $\Phi_{e}$ includes the magnetic and Josephson components and can be written as

$$
U\left(\phi, \phi_{e}\right)=E_{L}\left[0.5\left(\phi-\phi_{e}\right)^{2}-\beta_{L}(1+\cos \phi)\right],
$$

where $E_{L}=\left(\Phi_{0} / 2 \pi\right)^{2} / L=E_{J} / \beta_{L}$ is characteristic magnetic energy associated with the loop inductance, the Josephson coupling energy $E_{J}=\left(\Phi_{0} / 2 \pi\right) I_{c}$, the phase variable $\phi$ and the phase bias $\phi_{e}=2 \pi \Phi_{e} / \Phi_{0}$. For smal values of $\beta_{L} \ll 1$, the potential Eq. (1) yields the almost parabolic shape of the global single well (first term in Eq. (11)), whereas for the values $\beta_{L}$ appreciably greater that 1 , the series of wells are superimposed on the globa. parabola, so the bottom parts of these local minima car. also be approximated by the quadratic parabolas. In the case of large density of the levels within these parabolas. the energy spectrum is also close to that of a harmonic oscillator. So, neither of these cases allows significant anharmonicity necessary for the efficient qubit operation.

The essentially different shape of the potential Eq. (1) with $\phi_{e}=\pi$ is, however, achieved for $\beta_{L} \approx 1$, i.e. when the quadratic magnetic term is partially compensated by the quadratic term in the Josephson energy expansion near the bottom $(U=0)$ of the single well centered at $\varphi \equiv \phi-\pi=0$, i.e.

$$
U(\varphi) \approx E_{J}\left[-\frac{\left(\beta_{L}-1\right)}{2 \beta_{L}} \varphi^{2}+\frac{1}{24} \varphi^{4}+O\left(\varphi^{6}\right)\right] .
$$

In the ultimate case of $\beta_{L}=1$, Eq. (2) provides the obtuse shape of the quartic parabola. Taking into account the finite kinetic energy of the system in corresponding Schrödinger equation, $\hat{Q}^{2} / 2 C=-4 E_{c} \partial_{\phi \phi}$, where $\hat{Q}=-i(2 e) \partial_{\phi}$ is the charge operator [23], one can perform quantization of the system. Application of the quasiclassical quantization rule of Bohr-Sommerfeld yields for this quartic oscillator the energy levels obeying the
4/3 power law [24]:

$$
E_{n}^{(\mathrm{qc})}=\epsilon(n+1 / 2)^{4 / 3},
$$

with prefactor $\epsilon$ which in terms of the parameters of our circuit is equal to

$$
\epsilon=2^{-5 / 3} 3[\pi / \mathrm{K}(1 / 2)]^{4 / 3}\left(E_{J} E_{c}^{2}\right)^{1 / 3} \approx 1.9\left(E_{J} E_{c}^{2}\right)^{1 / 3},
$$

where $\mathrm{K}(k)$ is the complete elliptic integral of the first kind. Thus, the energy spectrum in the quartic potential takes intermediate position between the equidistant spectrum of the harmonic oscillator $E_{n} \propto(n+1 / 2)$ and that of the rectangular well, $E_{n} \propto(n+1)^{2}$, having extremely high anharmonicity. Expressions (3) and (4) are exact for the higher levels $(n \gg 1)$ and large "mass" (capacitance $C$ ), ensuring the very large ratio of the Josephson energy $E_{J}$ to the charging energy $E_{c}=e^{2} / 2 C$. An estimate of the anharmonicity factor in this quasiclassical approximation can be immediately obtained from Eq. (3):

$$
\delta_{\mathrm{qc}}=\left(\Delta E_{1}-\Delta E_{0}\right) / \Delta E_{0} \approx 26 \% .
$$

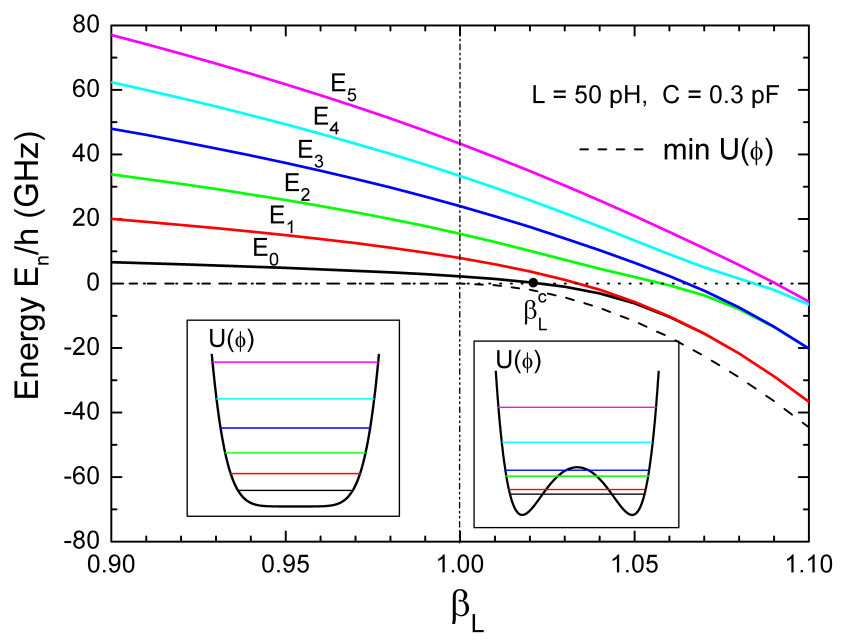

FIG. 2: (Color online) Position of the lowest six levels (solid lines) in the potential Eq. (1) for $\phi_{e}=\pi$ as a function of parameter $\beta_{L}$ for typical values of $L$ and $C$, yielding $E_{J} / E_{c} \sim$ $E_{L} / E_{c} \approx 5.1 \times 10^{4}$. With an increase of $\beta_{L}$, the spectrum crosses over from that of the harmonic oscillator type (left inset) to the set of the doublets (right inset), corresponding to the weak coupling of the oscillator-type states in two separate wells. The spectrum in the central region $\beta_{L} \approx 1$ is strongly anharmonic. The dashed line shows the bottom energy of the potential $U\left(\phi, \phi_{e}=\pi\right)$, which in the case of $\beta_{L}>1$ is equal to $-\Delta U \approx-1.5 E_{L}\left(\beta_{L}-1\right)^{2} / \beta_{L}$ (in other words, $\Delta U$ is the height of the energy barrier in the right inset) [20, 21]. The dotted (zero-level) line indicates the energy in the symmetry point $\phi=0$, i.e. at the bottom of the single well $\left(\beta_{L} \leq 1\right)$ or at the top of the energy barrier $\left(\beta_{L}>1\right)$. The black dot shows the critical value $\beta_{L}^{c}$ at which the ground state energy level touches the top of the barrier separating the two wells. 
The numerical solution of the corresponding Schrödinger equation with potential energy Eq. (1) yields in the limit $E_{J} / E_{c} \gg 1$ an even larger value of the anharmonicity factor, $\delta \approx 33 \%$ (see the energy spectrum in Fig. 2). These values substantially exceed the typical anharmonicity values of the conventional phase qubit, $\left|\delta_{\text {phase }}\right| \approx 3 \%$, for the number of levels inside the cubic-parabola well equal to four [2, 6], and transmon-qubit, $\left|\delta_{\text {transmon }}\right| \approx$ $\left(E_{c} / 8 E_{J}\right)^{1 / 2} \lesssim 5 \%$ for optimum values $E_{J} / E_{c} \gtrsim 50[12$ ]. Moreover, in contrast to the negative values of $\delta$ in these examples, the series of the energy levels in the quartic potential has positive $\delta>0$, i.e. corresponds to successively increasing level spacings $\Delta E_{1}<\Delta E_{2}<\Delta E_{3} \ldots$

Such a large, positive anharmonicity is a great advantage of the quartic potential qubit allowing manipulation within the two basis qubit states $|0\rangle$ and $|1\rangle$ not only when applying resonant microwave field, $\nu_{\mu \mathrm{w}} \approx \nu_{10}$, but also when applying control microwave signals with large frequency detuning or using rather wide-spectrum rectangular-pulse control signals. The characteristic qubit frequency $\nu_{10}=\Delta E_{0} / h$ and the anharmonicity factor $\delta$ computed from the Schrödinger equation for the
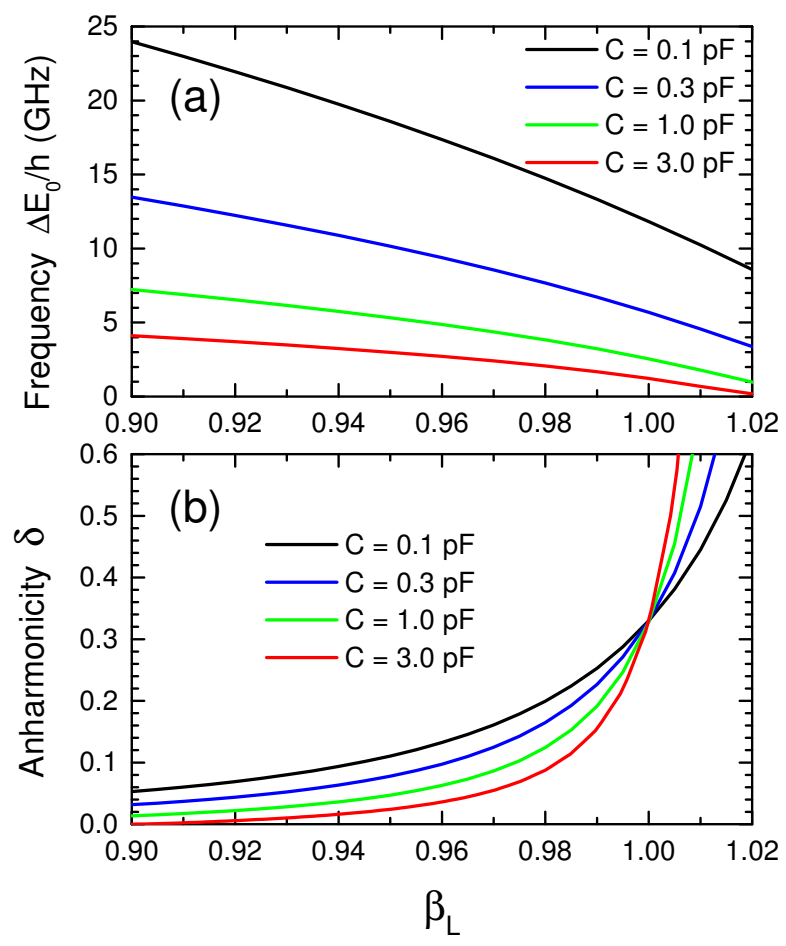

FIG. 3: (Color online) (a) The qubit frequency as a function of parameter $\beta_{L}$ for fixed $L=50 \mathrm{pH}$ and several values of capacitance $C=0.1,0.3,1.0$ and $3.0 \mathrm{pF}$ (from top to bottom), corresponding to the values of the ratio $E_{L} / E_{c} \approx$ $1.7 \times 10^{4}, 5.1 \times 10^{4}, 1.7 \times 10^{5}$ and $5.1 \times 10^{5}$. (b) Anharmonicity parameter $\delta$ as a function of parameter $\beta_{L}$ for the same as in (a) inductance $L$ and capacitance values (from top to bottom). original potential Eq. (1) in the range $0.9 \leq \beta_{L} \leq 1.02$ are shown in Fig. 3. One can see that the significant range in the tuning of the qubit frequency within the range of sufficiently large anharmonicity $(\sim 20-50 \%)$ is attained at a rather fine (typically $\pm 1-2 \%$ ) tuning of $\beta_{L}$ around the value $\beta_{L}=1$. Such tuning of $\beta_{L}$ is possible in the circuit having the compound configuration shown in Fig. 1b. For values of $\beta_{L}>1$, the symmetric energy potential has two minima and a barrier between them. The position of the ground state level depends on $\beta_{L}$ and the ratio of the characteristic energies $E_{J} / E_{c}=\beta_{L} E_{L} / E_{c}$. The value of $\beta_{L}$ at which the ground state level touches the top of the barrier sets the upper limit $\beta_{L}^{c}$ for the quartic qubit (marked in Fig.2 by solid dot). At $\beta_{L}>\beta_{L}^{c}$, the qubit energy dramatically decreases and the qubit states are nearly the symmetric and antisymmetric combinations of the states inside the two wells (see the right inset in Fig. 2). Although the qubit with such parameters has very large anharmonicity and can be nicely controlled by dc flux pulses [21, 22], its readout can hardly be accomplished in a dispersive fashion.

Another advantage of the phase qubit having the energy potential of the shape close to the quartic one is a strong dependence of its Josephson inductance $L_{J}\left(\Phi_{e}, n\right)$ on the quantum state $|n\rangle$. The observed value of the reverse inductance is related to the local curvature of the dependence of corresponding energy $E_{n}$ on flux $\Phi_{e}$ (see, e.g., Ref.[14]),

$$
L_{J}^{-1}\left(\Phi_{e}, n\right)=\frac{2 \pi}{\Phi_{0}}\left\langle n\left|\frac{\partial \hat{I}\left(\phi, \phi_{e}\right)}{\partial \phi_{e}}\right| n\right\rangle=\frac{\partial^{2} E_{n}\left(\Phi_{e}\right)}{\partial \Phi_{e}^{2}},
$$

where $\hat{I}$ is the operator of supercurrent circulating in the

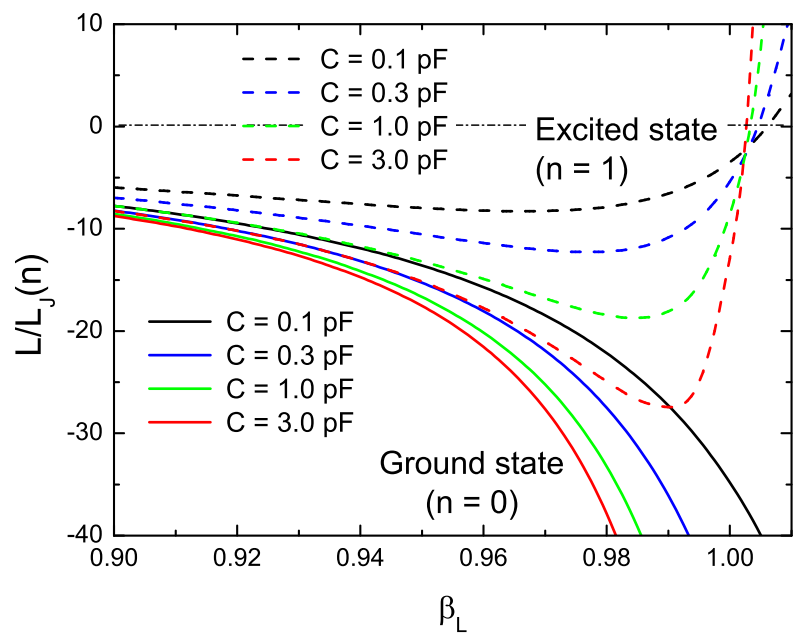

FIG. 4: (Color online) The values of the Josephson inductance of the quartic potential qubit in the ground (solid lines) and excited (dashed lines) states calculated for the geometric inductance value $L=50 \mathrm{pH}$ and the set of capacitances $C$, increasing from top to bottom for both groups of curves. 
qubit loop. The dependence of the reverse inductance $L_{J}\left(\Phi_{e}=\Phi_{0} / 2, n\right)$ calculated numerically in the two lowest quantum states $(n=0$ and 1$)$ for $L=50 \mathrm{pH}$ and the same set of capacitances $C$ as in Fig. 3 is shown in Fig.4. One can see that the ratio of the geometrical to Josephson inductances $L / L_{J}$ takes large and very different values that can be favorably used for the dispersive readout, ensuring a sufficiently large output signal. Note that for $\beta_{L}<1$, both inductances $L_{J}(n=0)$ and $L_{J}(n=1)$ are negative, whereas at $\beta_{L}>1$ the inductance $L_{J}(n=1)$ changes the sign to positive.

The readout of this qubit is based on the measurements of the reactive part (inductance) of the loop impedance probed by a low-frequency ac signal, $f \ll \nu_{10}$ of sufficiently small amplitude [25] (see also Ref. [10]). This signal is supplied by a rf-driven oscillator (Fig. 1a) as an alternating biasing flux, $\Phi_{e}=0.5 \Phi_{0}+M I_{L}$ or $\phi_{e}=$ $\pi+\delta \phi_{e}$, where $\delta \phi_{e}=a \cos (2 \pi f t)$ with $a \ll 1$. Here $M=\kappa\left(L L_{R}\right)^{1 / 2}$ is mutual inductance, $\kappa$, a dimensionless coupling coefficient and $I_{L}$, the ac current in inductance $L_{R}$. Coupling of the qubit to the resonance tank circuit causes renormalization of the circuit inductance (see, e.g. [10, 14]),

$$
L_{R}^{(n)}=L_{R}\left(1-\kappa^{2} L / L_{J}(n)\right)
$$

and the resonance frequencies $\omega_{n}=\left[L_{R}^{(n)} C\right]^{-1 / 2}$, where $n=0$ and 1 . The relative difference of the resonance frequencies for the qubit in the excited and ground states is

$$
\frac{\delta \omega}{\omega_{0}}=\frac{\omega_{1}-\omega_{0}}{\omega_{0}}=\sqrt{\frac{1-\kappa^{2} L / L_{J}(0)}{1-\kappa^{2} L / L_{J}(1)}}-1 .
$$

Figure 5] shows this relative frequency shift versus parameter $\beta_{L}$. One can see that for the rather conservative value of dimensionless coupling $\kappa=0.05$, the relative frequency shift can achieve the easily measured values of about $10 \%$. The efficiency of the dispersive readout can be improved in the non-linear regime with bifurcation [18]. With our device this regime can be achieved in the resonance circuit including, for example, a Josephson junction (marked in the diagram in Fig. 1 by a dashed cross). Due to the high sensitivity of the amplitude (phase) bifurcation to the threshold determined by the effective resonance frequency of the circuit, one can expect a readout with high fidelity even at a rather weak coupling of the qubit and the resonator (compare with the readout of quantronium in Ref. [26]). Further improvement of the readout can be achieved in the QED-based circuit including this qubit [27].

The loop configuration and frequency detuning of the quartic qubits should allow their inductive coupling with variable strength keeping both qubits in optimal points. Variable coupling of the optimally biased qubit to a superconducting resonator is also possible. More sophisticated coupling of the pairs of quartic qubits can be

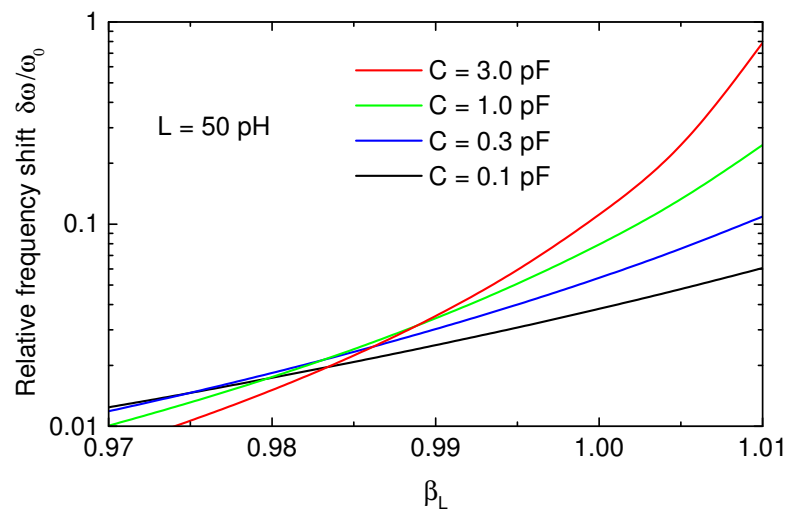

FIG. 5: (Color online) The resonance frequency shift in the circuit due to excitation of the qubit with the inductance value $L=50 \mathrm{pH}$ and the set of capacitances $C$, decreasing from top to bottom. The dimensionless coupling coefficient $\kappa=0.05$.

accomplished, for example, using a Josephson-junction coupler in a fashion recently proposed by Harris et al. [28].

In conclusion, we have shown that the phase qubit of the rf-SQUID configuration with parameter $\beta_{L} \approx 1$ and flux bias $\Phi_{e}=\Phi_{0} / 2$ has remarkable characteristics. Still, we expect that implementation of this qubit requires the solution of several experimental problems. For example, due to a high sensitivity of the qubit parameters to the magnitude of $\beta_{L}$, whose optimum values lie within a rather narrow range $( \pm 1-2 \%)$, particular precaution should be taken against fluctuations in the line controlling the effective Josephson coupling in the circuit (see Fig. 1b), because otherwise it may cause significant dephasing of the qubit. Furthermore, flux bias $\Phi_{e}=\Phi_{0} / 2$ should also be set as precisely as possible. Experimentally, it can be realized either by freezing the flux $\Phi_{e}=\Phi_{0}$ in the main loop having a symmetric gradiometer configuration 29], or by including in the loop of a Josephson $\pi$-junction [30] with a sufficiently high critical current ensuring the steady phase shift of $\pi$.

Of course, similar to properties of the conventional types of the phase qubits, the coherence characteristics of the quartic qubit will be strongly dependent on the material properties of the circuit. Minimizing the losses due to the qubit coupling to microscopic degrees of freedom (two-level systems) inside the dielectrics surrounding the superconducting circuit (the substrate, insulator inside the capacitor, the junction barriers, etc.) play crucial role for improving the qubit coherence [4]. Since the operation and tuning of the quartic qubit is possible without leaving the optimal point, one may expect a weaker coupling of the qubit to these microscopic two-level systems located inside dielectrics and, therefore, a better quantum coherence. Moreover, the zero persistent supercurrent circulating in the qubit loop at the optimum 
bias, $\phi_{e}=\pi$, may also reduce the effect of quasiparticle tunneling on the qubit coherence. Probably, such weakening of the qubit coupling to external degrees of freedom can explain reasonably good coherence characteris$\operatorname{tics}\left(\tau_{\text {Rabi }} \approx 60 \mathrm{~ns}\right)$ of the $\mathrm{Nb}$ camelback qubit operated in an optimal point at zero persistent current [16]. Anyway, the properties of the proposed quartic qubit will be clarified in experiment which is currently in the preparation stage.

We thank Michael Wulf and Ralf Dolata for useful discussions. This work was partially supported by the EU through the EuroSQIP project and DFG (German Science Foundation) through grant ZO124/2-1.

[1] Yu. Makhlin, G. Schön, and A. Shnirman, Rev. Mod. Phys. 73, 357 (2001).

[2] M. H. Devoret and J. M. Martinis, Quantum Information Processing 3, 163 (2004).

[3] G. Wendin and V. S. Shumeiko, in: Handbook of Theoretical and Computational Nanotechnology, ed. M. Rieth and W. Schommers (ASP, Los Angeles) 3, 223 (2006).

[4] J. M. Martinis, Quantum Information Processing 8, 81 (2009).

[5] J. M. Martinis, S. Nam, J. Aumentado and C. Urbina, Phys. Rev. Lett. 89, 117901 (2002).

[6] M. Steffen, J. M. Martinis, and I. L. Chuang, Phys. Rev. B 68, 224518 (2003).

[7] E. Lucero, M. Hofheinz, M. Ansmann, R. C. Bialczak, N. Katz, M. Neeley, A. D. O'Connell, H. Wang, A. N. Cleland and J. M. Martinis, Phys. Rev. Lett. 100, 247001 (2008).

[8] Y. Nakamura, Yu. A. Pashkin and J. S. Tsai, Nature 398, 786, (1999).

[9] D. Vion, A. Aassime, A. Cottet, P. Joyez, H. Pothier, C. Urbina, D. Esteve and M. H. Devoret, Science 296, 886 (2002).

[10] A. B. Zorin, Physica C (Amsterdam) 368, 284 (2002).

[11] I. Chiorescu, Y. Nakamura, C. J. P. M. Harmans and J. E. Mooij, Science 299, 1869 (2003).

[12] J. Koch, T. M. Yu, J. Gambetta, A. A. Houck, D. I. Schuster, J. Majer, A. Blais, M. H. Devoret, S. M. Girvin, and R. J. Schoelkopf, Phys. Rev. A 76, 042319 (2007).

[13] V. E. Manucharyan, J. Koch, L. I. Glazman and M. H. Devoret, arXiv:0906.0831.

[14] A. B. Zorin, JETP 98, 1250 (2004).

[15] F. Chiarello, M. G. Castellano, G. Torrioli, S. Poletto, C. Cosmelli, P. Carelli, D. V. Balashov, M. I. Khabipov, and A. B. Zorin, Appl. Phys. Lett. 93, 042504 (2008).

[16] E. Hoskinson, F. Lecocq, N. Didier, A. Fay, F. W. J. Hekking, W. Guichard, O. Buisson, R. Dolata, B. Mackrodt, and A. B. Zorin, Phys. Rev. Lett. 102, 097004 (2009).

[17] A. Lupaşcu, C. J. M. Verwijs, R. N. Schouten, C. J. P. M. Harmans, and J. E. Mooij, Phys. Rev. Lett. 93, 177006 (2004).

[18] I. Siddiqi, R. Vijay, F. Pierre, C. M. Wilson, M. Metcalfe, C. Rigetti, L. Frunzio, and M. H. Devoret, Phys. Rev. Lett. 93, 207002 (2004).

[19] A. Lupaşcu, E. F. C. Driessen, L. Roschier, C. J. P. M. Harmans, and J.E. Mooij, Phys. Rev. Lett. 96, 127003 (2006).

[20] F. Chiarello, Phys. Lett. A 277, 189 (2000).

[21] F. Chiarello, Eur. Phys. J. B 55, 7 (2007).

[22] S. Poletto, F. Chiarello, M. G. Castellano, J. Lisenfeld, A. Lukashenko, C. Cosmelli, G. Torrioli, P. Carelli and A. V. Ustinov, New J. Phys. 11, 013009 (2009).

[23] P. W. Anderson, in Lectures on many-body problem, ed. E. R. Caianiello (Academic Press, NY) 2, 113 (1964).

[24] see, for example, A. M. Sánchez and J. D. Bejarano, J. Phys. A: Math. Gen. 19, 887 (1986) and references therein.

[25] R. Rifkin and B. S. Deaver, Jr., Phys. Rev. B 13, 3894 (1976).

[26] I. Siddiqi, R. Vijay, M. Metcalfe, E. Boaknin, L. Frunzio, R. J. Schoelkopf and M. H. Devoret, Phys. Rev. B 73, 054510 (2006).

[27] M. B. Metcalfe, E. Boaknin, V. Manucharyan, R. Vijay, I. Siddiqi, C. Rigetti, L. Frunzio, R. J. Schoelkopf, and M. H. Devoret, Phys. Rev. B 76, 174516 (2007).

[28] R. Harris, T. Lanting, A. J. Berkley, J. Johansson, M. W. Johnson, P. Bunyk, E. Ladizinsky, N. Ladizinsky, T. Oh, and S. Han, Phys. Rev. B 80, 052506 (2009).

[29] J. B. Majer, J. R. Butcher and J. E. Mooij, Appl. Phys. Lett. 80, 3638 (2002).

[30] L. B. Ioffe, V. B. Geshkenbein, M. V. Feigel'man, A. L. Fauchŕe and G. Blatter, Nature 398, 679 (1999). 